\title{
High prevalence of complementary and alternative medicine use in patients with genetically proven mitochondrial disorders
}

\author{
Sebastian Franik • Hidde H. Huidekoper • Gepke Visser • Maaike de Vries • \\ Lonneke de Boer • Marion Hermans-Peters • Richard Rodenburg • Chris Verhaak • \\ Arine M. Vlieger • Jan A. M. Smeitink • Mirian C. H. Janssen • Saskia B. Wortmann
}

Received: 8 July 2014 / Revised: 10 September 2014 / Accepted: 16 September 2014 / Published online: 11 October 2014

(C) SSIEM 2014

\begin{abstract}
Despite major advances in understanding the pathophysiology of mitochondrial diseases, clinical management of these conditions remains largely supportive, and no effective treatment is available. We therefore assumed that the burden of disease combined with the lack of adequate treatment leaves open a big market for complementary and alternative medicine use. The objective of this study was to evaluate the use and perceived effectiveness of complementary and alternative medicine in children and adults with genetically proven mitochondrial disease. The reported use was surprisingly high, with $88 \%$ of children and $91 \%$ of adults having used some kind of complementary and alternative medicine in the last 2 years. Also, the mean cost of these treatments was impressive, being $€ 489 /$ year for children and $€ 359 /$ year for adult patients. Over-the-counter remedies (e.g., food supplements, homeopathy) and self-help techniques
\end{abstract}

Communicated by: Eva Morava

Electronic supplementary material The online version of this article (doi:10.1007/s10545-014-9773-9) contains supplementary material, which is available to authorized users.

S. Franik $\cdot$ M. de Vries $\cdot$ L. de Boer $\cdot$ M. Hermans-Peters ·

R. Rodenburg $\cdot$ C. Verhaak · J. A. M. Smeitink • M. C. H. Janssen •

S. B. Wortmann $(\triangle)$

Nijmegen Centre for Mitochondrial Disorders (NCMD) at the

Amalia Children's Hospital, Radboudumc, P.O. Box 9101,

6500 HB Nijmegen, The Netherlands

e-mail: saskia.wortmann-hagemann@radboudumc.nl

H. H. Huidekoper

Department of Pediatrics, University of Amsterdam, Academic

Medical Center, Amsterdam, The Netherlands

G. Visser

Department of Paediatric Gastroenterology and Metabolic Diseases,

Wilhelmina Children's Hospital, UMC Utrecht, Utrecht,

The Netherlands (e.g., Reiki, yoga) were the most frequently used complementary and alternative therapies in our cohort: $54 \%$ of children and $60 \%$ of adults reported the various complementary and alternative medicine therapies to be effective. Given the fact that currently no effective treatment exists, further research toward the different therapies is needed, as our study clearly demonstrates that such therapies are highly sought after by affected patients.

\section{Introduction}

Disorders of the mitochondrial oxidative phosphorylation (OXPHOS) system are rare disorders that can present with virtually any clinical symptom. Underlying mutations can be found in nuclear genes as well as in the maternally inherited

C. Verhaak

Department of Psychology, Radboudumc, Nijmegen,

The Netherlands

A. M. Vlieger

St Antonius Ziekenhuis, Nieuwegein, The Netherlands

M. C. H. Janssen

Department of Internal Medicine, Radboudumc, Nijmegen,

The Netherlands 
mitochondrial genome (Taylor and Turnbull 2005; Koopman et al. 2012; DiMauro et al. 2013). Despite major advances in understanding the pathophysiology of mitochondrial diseases, clinical management remains largely supportive, and no effective treatment is available (Pfeffer et al. 2013). Often, highdose multivitamin cocktails are prescribed to patients, but there are no studies supporting the effectiveness, with the exception of some reported cases of coenzyme Q10 deficiency responding to therapy (Emmanuele et al. 2012).

Complementary and alternative medicine (CAM) refers to a group of diverse medical health care systems, practices, and products that are not considered to be part of conventional medicine. Renowned therapies, such as homeopathy, acupuncture, yoga, and herbal supplements, fall within this spectrum of therapies. They can be assigned to four major groups: alternative medical systems, mind-body interventions, biologically based therapies, and energy therapies (National Center for Complementary and Alternative Medicine: What are the major types of complementary and alternative medicine? Retrieved from: http://nccam.nih.gov/health/whatiscam; NCCAM 2013). Once such a treatment modality is used alongside conventional therapy, it is defined as complementary, whereas that same therapy is called alternative when it substitutes mainstream care.

In recent years, CAM therapies have become quite popular, with approximately one third of all adult and pediatric patients using CAM (Madsen et al. 2003; Tindle et al. 2005; Vlieger et al. 2006). There is growing interest in the use of CAM in different pediatric and adult patient populations, especially in pediatric oncology patients, where usage ranges between 20 and $60 \%$ are reported (Bishop et al. 2010; Singendonk et al. 2013). For these patient groups, evidence-based treatment protocols exist and have shown such therapies to be effective, which is in striking contrast to the group of patients with mitochondrial disorders. We therefore assume that the burden of disease combined with the lack of adequate treatment for mitochondrial disorders leaves open a big market for CAM due to the helplessness of affected patients. The objective of this study was to evaluate the use and perceived effectiveness of CAM in children and adults with genetically proven mitochondrial disease.

\section{Methods}

Patients

All adult patients and caretakers of pediatric patients with a genetically proven diagnosis of a mitochondrial disorder regularly attending the outpatient clinics of Pediatrics or Internal Medicine at the Nijmegen Centre for Mitochondrial Disorders (NCMD) received an informational letter and questionnaire by postal service. (Caretakers of) patients with an insufficient knowledge of the Dutch language were not recruited. Patients with the mitochondrial m.3243A $>$ G mutation were recruited for a separate study. Patients attending mainly other specialists [e.g., patients with Leber's hereditary optic neuropathy (LHON) or chronic progressive external ophthalmoplegia (CPEO) phenotype attending the ophthalmology department] were not included.

\section{Study design}

This is a single-center study investigating the prevalence of CAM use by means of a questionnaire consisting of 21 questions, requiring approximately $20 \mathrm{~min}$ to complete. The instrument was modified from a previous Dutch study assessing CAM use among pediatric oncology patients and was filled in anonymously by adult patients or by caretakers of pediatric patients (referred to as children or pediatric patients in the further course of this manuscript) (Singendonk et al. 2013). The questionnaire was approved by the ethics committee of the Radboud UMC Nijmegen (Comissie mensgebonden onderzoek, CMO Arnhem-Nijmegen). It contained questions about the patient's gender, age, and ethnic background. From the four major domains in CAM as defined by the National Center for Complementary and Alternative Medicine, the 16 most popular modalities in The Netherlands were listed (NCCAM 2013). Additionally, patients could mention other, not listed, CAM therapies. They were asked about any CAM therapies they had used in the past 2 years, their reasons for using it, the costs of the treatment, and the perceived effectiveness of the modalities used. CAM therapies were subdivided into three categories: over-the-counter remedies, self-help techniques, and therapies as prescribed by a CAM therapist/physician. Additionally, communication with the treating metabolic physician regarding CAM use was evaluated, and participants were asked about the desirability of advice on and the availability of CAM modalities in the hospital setting. Finally, patients were asked about the necessity of research on CAM therapies and possible willingness to participate in future clinical trials.

\section{Results}

In total, questionnaires were send to 38 pediatric and 46 adult patients; 24 (63\%) and 33 (72\%) questionnaires, respectively, were returned. As some questionnaires were only partially completed, our analyses report different numbers per question. Results are rounded to integer. Demographic and disease baseline characteristics are shown in Table 1. Patient age ranged from 2 to 16 (median 8.6) years in pediatric and from 19 to 66 (median 41.7) years in adult patients. Gender was 
Table 1 Demographics, disease baseline characteristics, and use of different complementary and alternative medicine (CAM) therapies among patients with mitochondrial disorders

\begin{tabular}{lcc}
\hline & Children $(N=24) ; n(\%)$ & Adults $(N=33) ; n(\%)$ \\
\hline Median age in years (range) & $8.6(2-16)$ & $41.7(19-66)$ \\
Male & $16(67)$ & $16(48)$ \\
Time since diagnosis & & \\
$<1$ year & $4(16.7)$ & $3(9.09)$ \\
$1-5$ years & $8(33.3)$ & $10(30.30)$ \\
$5-10$ years & $8(33.3)$ & $9(27.27)$ \\
$>10$ years & $4(16.7)$ & $11(33.33)$ \\
All types of CAM & $21(88)$ & $30(91)$ \\
Over-the-counter remedies & $19(79)$ & $25(76)$ \\
Food supplements & $15(63)$ & $23(70)$ \\
Homeopathy & $12(50)$ & $9(27)$ \\
Herbal remedies & $5(21)$ & $0(0)$ \\
Other & $5(21)$ & $4(12)$ \\
Self-help techniques & $8(33)$ & $14(42)$ \\
Massage or Reiki & $5(21)$ & $6(18)$ \\
Others & $3(12.50)$ & $4(12)$ \\
Relaxation exercise & $2(8)$ & $2(6)$ \\
Meditation or yoga & $1(4)$ & $6(18)$ \\
Aroma therapy & $1(4)$ & $6(18)$ \\
Visualization or hypnosis & $0(0)$ & $3(3)$ \\
Healing ceremony & $0(0)$ & $0(0)$ \\
\hline
\end{tabular}

equally distributed amongst adult patients; in the pediatric population, boys dominated (67\%).

\section{CAM use and costs}

Eighty-eight percent of children (C) and $91 \%$ of adults (A) used at least one type of CAM therapy within the 2 years prior to the study. The most common type both in children and adults was over-the-counter remedies (C $79 \%$, A $76 \%$ ), with food supplements being the most popular (C $63 \%$, A $70 \%$ ), followed by various products of homeopathic origin (C $50 \%$, A $27 \%$ ). Food supplements were the only CAM therapies reported to be prescribed by a metabolic physician and were prescribed for $73 \%$ of children and $60 \%$ of adults using them. Self-help techniques were reported to be used frequently by both children (33\%) and adults (42\%), most commonly massage or Reiki (C $21 \%$, A $18 \%$ ), and in adults also meditation or yoga (each $18 \%$ ); $38 \%$ of children and $21 \%$ of adults had visited a CAM therapist within the prior 2 years. For children, homeopathy (25\%) and manual-based therapies (21\%) were prescribed most frequently by the consulted CAM therapist, followed by phytotherapy $(17 \%)$. Adults most commonly were advised to use manual-based therapy (21\%), phytotherapy (9\%), and Chinese medicine, including acupuncture $(6 \%)$. Among patients using CAM, $46 \%$ of children and $29 \%$ of adults already used CAM therapy before diagnosis of their mitochondrial disease; $31 \%$ (A $29 \%$ ) started within 1 year after diagnosis, and $23 \%$ (A $43 \%$ ) $>1$ year after diagnosis. Patients had spent $€ 0-1,000$ on CAM per year [mean €489/year (C) and €359/year (A)].

\section{Perceived effectiveness}

The combined analysis of all different types of CAM shows that $54 \%$ of children and $60 \%$ of adults reported the various CAM therapies to be very or somewhat effective (Table 2); $21 \%$ of children and $17 \%$ of adults reported them to be ineffective; the remainder were unsure. The group of self-help techniques yielded the highest percentage of children and adults rating the therapy as very effective (C $55 \%$, A $67 \%$ ). A detailed analysis of the particular types of CAM therapies used is shown in Supplementary Tables 1 (C) and 2 (A).

\section{Reasons for CAM use}

All participants were asked if they used CAM therapy for a specific symptom associated with mitochondrial disease or to improve general well-being. They were able to choose between four given options: tiredness or low energy, muscle pain or cramps, general well-being, or other. The most reported reason in children was to improve general well-being (44\%); adults reported tiredness or low energy (38\%) to be the most important reason. The primary motivation for children was to improve the immunity and general condition (17\%), followed 
Table 2 Perceived effectiveness of complementary and alternative medical (CAM) therapies among patients with mitochondrial disorders

\begin{tabular}{|c|c|c|c|c|}
\hline & $\begin{array}{l}\text { Over-the-counter } \\
\text { remedies, } n(\%)\end{array}$ & $\begin{array}{l}\text { Self-help techniques, } \\
n(\%)\end{array}$ & $\begin{array}{l}\text { Prescribed by CAM physician/ } \\
\text { therapist, } n(\%)\end{array}$ & $\begin{array}{l}\text { All types of CAM, } \\
n(\%)\end{array}$ \\
\hline Children (n) & 35 & 11 & 17 & 63 \\
\hline Very effective & $7(20)$ & $6(55)$ & $11(65)$ & $24(38)$ \\
\hline Somewhat effective & $9(26)$ & $1(9)$ & $0(0)$ & $10(16)$ \\
\hline Not effective & $9(26)$ & $1(9)$ & $3(18)$ & $13(21)$ \\
\hline Unknown & $10(29)$ & $3(27)$ & $3(18)$ & $16(25)$ \\
\hline Adults $n$ & 60 & 24 & 11 & 95 \\
\hline Very effective & $6(10)$ & $16(67)$ & $4(36)$ & $26(27)$ \\
\hline Somewhat effective & $20(33)$ & $5(21)$ & $5(45)$ & $30(32)$ \\
\hline Not effective & $13(22)$ & $1(4)$ & $2(18)$ & $16(17)$ \\
\hline Unknown & $21(35)$ & $2(8)$ & $0(0)$ & $23(24)$ \\
\hline
\end{tabular}

by suggested by family or friends (13\%) and the wish to get better $(10 \%)$. Among adults, the wish to get better and to improve immunity and general condition was equally reported, with $24 \%$ each as their primary motivations, followed by more control over own life and disease (19\%).

Communication with treating metabolic physician

About one third of children (33\%) and slightly more adults (38\%) stated that they had communicated their CAM use with their treating metabolic physician. The most common response of the metabolic physician was positive both for children $(63 \%)$ and adults (100\%) or neutral $(25 \%$ and $0 \%$, respectively). Communication was always (C $100 \%$ ) or most commonly (A $67 \%$ ) initiated by the patient.

Attitudes towards research

Future research about CAM therapies was rated as important or very important by $87 \%$ of children and $81 \%$ of adults. Subsequently, $48 \%$ of children and $80 \%$ of adults were willing to participate in a future trial. Advice about CAM therapies by the treating metabolic physician was also rated important by both children (83\%) and adults (89\%).

\section{Discussion}

In recent years, CAM therapies have become popular, with approximately one third of all adult and pediatric patients using CAM (Madsen et al. 2003; Tindle et al. 2005; Vlieger et al. 2006). Our study is the first to report on CAM use in children and adults with genetically proven mitochondrial disease. The reported use of CAM in our patient population was surprisingly high, with $88 \%$ of children and $91 \%$ of adults having used some type of CAM in the last 2 years
(Table 1). Also the mean paid for these treatments was impressive, being $€ 489 /$ year for children and $€ 359 /$ year for adults. The high percentage of CAM use found in our study is most likely due to the combination of lack of effective treatment for these diseases and of the disease having a great impact on the daily quality of life due to symptoms such as tiredness, low energy, and muscle pain and cramps, as reported by participants in our study. This helplessness and the lack of evidence-based alternatives leaves open a large market for non-evidence-based CAM use in patients with mitochondrial disease. Comparable rates of CAM use ( $~ 80 \%)$ have been reported in pediatric patients with Duchenne or Becker muscular dystrophy (Nabukera et al. 2012). For patients with lysosomal storage disorders, the use of CAM reported in one single-center study was lower: C $8 \% / \mathrm{A} 45 \%$ for type I Gaucher disease, C $50 \% / \mathrm{A} 41 \%$ of Fabry disease, and C $33 \% / A 47 \%$ of type B Niemann-Pick patients (Balwani et al. 2009). Due to the very limited number of studies and the small number of patients included in that study, the reasons for the differences in CAM use can only be speculative. Most likely, the most important reason is that, in contrast to our patient group, treatments are available for both Duchenne/Becker muscular dystrophy and for several lysosomal storage disorders, which are at least able to slow down the the progressive and disabling course of those diseases.

In our study, $54 \%$ of children and $60 \%$ of adults reported the various $\mathrm{CAM}$ therapies to be effective (very effective $\mathrm{C}$ $38 \%$, A $16 \%$, somewhat effective C $27 \%$, A $32 \%$; Table 2). Over-the-counter remedies (e.g., food supplements, homeopathy) and self-help techniques (e.g., Reiki, yoga) were the most frequently used (Table 1). Vitamin supplements such as riboflavin, coenzyme Q10, and thiamine, were not perceived as being effective by most patients (Supplementary Tables 1 and 2). This is an interesting finding and in line with studies that failed to prove the efficacy of vitamin supplements in mitochondrial disease (Pfeffer et al. 2013). Additionally, there is no consensus among physicians prescribing these vitamins 
(mostly as a mitochondrial cocktail) as to which supplements are most effective and at what dosages (Parikh et al. 2009, 2013). However, the Mitochondrial Medicine Society advises in their opinion statement that a therapeutic trial of coenzyme Q10, along with other antioxidants, should be attempted based on clinical experience and judgment (Parikh et al. 2009).

In contrast, the effectiveness of self-help techniques such as massage or yoga was rated positively (Supplementary Tables 1 and 2). We assume that the perceived benefits of self-help techniques are both physical and psychological, as patients feel that they can achieve muscular relaxation while positively influencing the course of disease. One should also take into account that patients paid up to $€ 1,000 /$ year for such therapies, and this might also improve the perceived level of effectiveness. It is difficult to compare data evaluating the perceived effectiveness with similar data for other disorders. Singendonk et al. (2013) reported the perceived effectiveness as being even higher: $75 \%$ of their study participants perceived CAM as effective (very effective $49 \%$, somewhat effective $26 \%$ ). There are no directly comparable data for an identical cohort of adult patients. The study evaluating CAM use in Duchenne patients did not report data on this issue, and for the lysosomal group, data were presented only per CAM therapy as a rating on a Likert scale, which makes a comparison impossible (Balwani et al. 2009, Nabukera et al. 2012).

In general, CAM is mostly considered to be "soft" and "natural" therapy by patients and often by their treating physician. However, CAM can have numerous side effects, ranging from mechanical injuries and infections by acupuncture needles, to contact dermatitis due to herbal remedies (Niggemann and Grüber 2003). Our study did not evaluate potential side effects of CAM. It is difficult to estimate the (long-term) side effects of vitamin supplements, but for the other most frequently used CAM therapy-self-helping techniques such as yoga or massage - no serious side effects are expected.

A strength of this study is the use of a validated, standardized questionnaire according to the recent European guidelines on standardization of CAM questionnaires, making it possible to compare the results of this study to previous studies using the same type of survey (Quandt et al. 2009). Since mitochondrial diseases are very rare and the genetic background is often not known, a study group of 57 patients including 24 children might be a representative cohort. However, being a single-center study is a limitation; therefore, the prevalence of and need for information, and attitude toward research reported by our participants, might be overestimated for the general population with mitochondrial disease. Furthermore, as this was a questionnaire-based study, respondent bias is likely to have an impact, possibly leading to over- or underestimation of treatment effects.
Given the lack of any effective treatment for mitochondrial disorders and the perceived effectiveness of CAM therapies reported in this study, more awareness and further research on this issue is needed. Accordingly, $87 \%$ of caretakers of children and $81 \%$ of adults rated further research on CAM therapies as important, and a high percentage (C $48 \%$, A $80 \%$ ) was willing to participate in future studies. Especially when taking into account that modern disease management is a continuous challenge that can only be successful when the patient and the treating physician are partners, further highquality, multicenter, randomized, and (if possible), doubleblind studies are needed to improve the quality of life for children and adults with mitochondrial disorders. Ideally, these should be performed in international collaboration and make use of standardized data collection in order to harmonize data and streamline research. To evaluate the effect of CAM (and other interventions) in upcoming treatment studies, standardized and validated questionnaires evaluating fatigue and quality of life (e.g., KIDSCREEN 52, Pediatric Evaluation of Disabilities Inventory, PedsQL Multidimensional Fatigue Scale) are available and should be used (Koene et al. 2013).

In conclusion, the prevalence of CAM use was very high ( $\sim 90 \%)$ in our cohort of children and adults with genetically proven mitochondrial disorders. The reported effectiveness for the different types of CAM therapy varied greatly, with self-help techniques rated most effective. Given the fact that no effective treatments exists for these disorders, further research into different CAM therapies is needed, and-as our study clearly demonstrates - is highly sought after by affected patients.

Acknowledgments We thank Jolanda Teunissen for excellent administrative assistance.

Conflict of interest None.

\section{References}

Balwani M, Fuerstman L, Desnick R, Buckley B, McGovern M (2009) Use of complementary and alternative medicine by patients with lysosomal storage diseases. Genet Med Off J Am Coll Med Genet 11(10):722-727

Bishop F, Prescott P, Chan Y, Saville J, von Elm E, Lewith G (2010) Prevalence of complementary medicine use in pediatric cancer: a systematic review. Pediatrics 125(4):768-776

DiMauro S, Schon EA, Carelli V, Hirano M (2013) The clinical maze of mitochondrial neurology. Nat Rev Neurol 9(8):429-444

Emmanuele V, López LC, López L et al (2012) Heterogeneity of coenzyme Q10 deficiency: patient study and literature review. Arch Neurol 69(8):978-983

Koene S, Jansen M, Verhaak CM, De Vrueh RL, De Groot IJ, Smeitink JA (2013) Towards the harmonization of outcome measures in children with mitochondrial disorders. Dev Med Child Neurol 55(8):698-706 
Koopman WJ, Willems PH, Smeitink JA (2012) Monogenic mitochondrial disorders. N Engl J Med 366(12):1132-1141

Madsen H, Andersen S, Nielsen RG, Dolmer BS, Høst A, Damkier A (2003) Use of complementary/alternative medicine among paediatric patients. Eur J Pediatr 162(5):334-341

Nabukera SK, Romitti PA, Campbell KA et al (2012) Use of complementary and alternative medicine by males with Duchenne or Becker muscular dystrophy. J Child Neurol 27(6):734-740

NCCAM (2013) National Center for Complementary and Alternative Medicine: What are the major types of complementary and alternative medicine? Accessed via http://nccam.nih.gov/health/whatiscam in June 2014

Niggemann B, Grüber C (2003) Side-effects of complementary and alternative medicine. Allergy 58(8):707-716

Parikh S, Saneto R, Falk MJ et al (2009) Curr Treat Options Neurol 11(6): 414-430

Parikh S, Goldstein A, Koenig MK et al (2013) Practice patterns of mitochondrial disease physicians in North America. Part 2: treatment, care and management. Mitochondrion 13(6):681-687
Pfeffer G, Horvath R, Klopstock T et al (2013) New treatments for mitochondrial disease-no time to drop our standards. Nat Rev Neurol 9(8):474-481

Quandt S, Verhoef M, Arcury T et al (2009) Development of an international questionnaire to measure use of complementary and alternative medicine (I-CAM-Q). J Altern Complement Med (New York, NY) 15(4):331-339

Singendonk M, Kaspers G-J, Naafs-Wilstra M, Meeteren A, Loeffen J, Vlieger A (2013) High prevalence of complementary and alternative medicine use in the Dutch pediatric oncology population: a multicenter survey. Eur J Pediatr 172(1):31-37

Taylor RW, Turnbull DM (2005) Mitochondrial DNA mutations in human disease. Nat Rev Genet 6(5):389-402

Tindle HA, Davis RB, Phillips RS, Eisenberg DM (2005) Trends in use of complementary and alternative medicine by US adults: 1997-2002. Altern Ther Health Med 11(1):42-49

Vlieger A, van de Putte E, Hoeksma H (2006) The use of complementary and alternative medicine in children at a general paediatric clinic and parental reasons for use. Ned Tijdschr Geneeskd 150(11):625-630 